\title{
Depression, ADHD, Job Stress and Sleep Problems with Dry Eye Disease in Korea
}

Kyong Jin Cho', Hong Kyu Kim¹, Myung Ho Lim²*, Hae Soon Baek ${ }^{3}$, Young Ae Yang ${ }^{4}$, Bong Hui Kang ${ }^{5}$, Jeong Yeob Lee ${ }^{6}$, Jeong Yun Kim ${ }^{7}$, Man Soo Kim ${ }^{8}$ and Chang Min Lee ${ }^{5}$

${ }^{1}$ Department of Opthalmology, College of Medicine, Dankook University, Cheonan, South Korea

${ }^{2}$ Department of Psychology, College of Social Science, Dankook University, Cheonan, South Korea

${ }^{3}$ Department of Nursing, Dankook University Hospital, Cheonan, South Korea

${ }^{4}$ Anseong Mental Health Center, Anseong, South Korea

${ }^{5}$ Department of Neurology, Dankook University, Cheonan, South Korea

${ }^{6}$ Good Morning Hospital, Seosan, South Korea

${ }^{7}$ Department of Biomedical Science, Graduate School, Dankook University, Cheonan, South Korea

${ }^{8}$ Catholic Institute for Visual Science; and Seoul St. Mary's Hospital, College of Medicine, The Catholic University of Korea, Seoul, Korea

\begin{abstract}
Purpose: For people with dry eye disease common problems in working area, the associations with depression, anxiety, attention deficit hyperactivity disorder (ADHD), job stress, and sleep problems. This study aims to examine the effects of dry eye disease and depression, anxiety, ADHD, job stress, and sleep problems through a self-rated questionnaire.

Subjects and Methods: Subjects included 139 people who complained of symptoms of dry eye disease for the first time between September 2014 and February 2015. The comparison group included 363 local adults without symptoms of dry eye disease. A psychiatric and ophthalmic questionnaire survey was given to the group having symptoms of dry eye disease. The presence of a significant difference in depression, anxiety, ADHD, job stress, and sleep problems between the dry eye disease group and the comparison group was evaluated, and each association was analyzed.
\end{abstract}

Results: The dry eye symptoms group showed significantly higher the Ocular Surface Disease Index (OSDI), Center for Epidemiological Studies-Depression Scale (CES-D), Korean Adult Attention Deficit Hyperactivity Disorder Scales (K-AADHS), and The Korean version of Pittsburgh Sleep Quality Index (PSQI-K) values than the comparison group $(p<0.001, p<0.001, p<0.001$, and $p<0.001)$. The result of the regression analysis indicated that depression symptoms and ADHD symptoms significantly increased the odd ratio of the dry eye disease symptoms group by 1.75 times and 2.18 times, respectively $(p=0.04$ and $p<0.001)$.

Conclusion: The dry eye symptoms group is accompanied by ADHD related issues as well as depression. Therefore, a psychiatric approach is needed along with physical treatment.

Keywords: Depression; ADHD; Job stress; Sleep problems; Dry eye disease

\section{Introduction}

Dry eye disease (DED) is a common ophthalmic disease in clinical applications, and its prevalence rate in the United States is approximately 5\%. The 2007 Report of the International Dry Eye Workshop report defined dry eye disease as a multifactorial disease of the tears and ocular surface that results in symptoms of discomfort, visual disturbance, and tear film instability with potential damage to the ocular surface. It is accompanied by increased osmolarity of the tear film and inflammation of the ocular surface" [1]. Despite its high prevalence rate, dry eye disease is difficult to cure because in many cases the examinations that are commonly used in clinical applications and the symptoms that patients complain of are inconsistent [2]. The diagnosis of dry eye disease is generally based on symptoms that patients complain of, which are the most important element for the diagnosis of dry eye disease [3]. There have been few studies on the association of dry eye disease and psychiatric symptoms or disorders [4]. Labbe et al. [5] performed various objective ophthalmic examinations for 1,456 ophthalmic patients, and reported that 241 patients had Dry eye disease and 138 patients showed depressive symptoms. They also reported that the Dry eye disease group had a significantly higher depression score than the control group. For 89 DES patients and 73 control group subjects, Li et al. [6] compared the associations of DES with depression and anxiety through the OSDI, the Self-rating Anxiety Scale (SAS), and
Self-rating Depression Scale (SDS) questionnaires. The results showed that the DES group had significantly higher anxiety and depression scores. In a study on 472 psychiatric patients with depressive and anxiety disorders, Wen et al. [7] reported that the prevalence rate of Dry eye disease was $13 \%$ (60 patients). In a large-scale epidemiologic survey on 2,454,458 subjects relevant to Veterans Affairs, Galor et al. [8] reported that $19 \%$ of the males and $22 \%$ of the females showed DES, and the risk factors of DES were post-traumatic stress disorder and depressive disorder. In a study on 6,655 adult females, $\mathrm{Na}$ et al. [9] reported that the clinical diagnosis group was $12.3 \%$ and the symptom group was $20.0 \%$, and the significant risk factors were severe psychological stress, depressive mood, anxiety/depression problems, and a history of psychological counseling. In a study on 248 ophthalmic patients who had visited the veterans Affairs Eye Clinic, Fernandez et al. [10] reported that the groups with PTSD and depression had significantly

*Corresponding author: Myung Ho Lim, Dandae Rho 119, Cheonan, South Korea, Tel: 82-41-550-3263; Fax: 82-41-550-3260; E-mail: paperose@dku.edu

Received June 10, 2015; Accepted September 28, 2015; Published October 05, 2015

Citation: Cho KJ, Kim HK, Lim MH, Baek HS, Yang YA, et al. (2015) Depression, ADHD, Job Stress, and Sleep Problems with Dry Eye Disease in Korea. J Psychiatry 18: 331 doi:10.4172/2378-5756.1000331

Copyright: ( $) 2015$ Cho KJ, et al. This is an open-access article distributed under the terms of the Creative Commons Attribution License, which permits unrestricted use, distribution, and reproduction in any medium, provided the original author and source are credited 
higher Dry eye disease scores than the groups without PTSD and depression, respectively. It has been a few reported that depression and anxiety, common internalized symptoms are related with the dry eye syndrome. However, there has been no report on the relationship of ADHD or ADHD symptoms, common externalized symptoms with dry eye syndrome. In this study, for the first time in Korea and any other country, the relationship between adult dry eye syndrome and ADHD symptom was examined, in addition to the relationship of dry eye syndrome with depression, anxiety, job stress, and sleep problems. ADHD is a common neurobehavioral disorder usually has its onset in early childhood, more rarely in adolescence. When ADHD persists into adulthood, the disorder may be of mild intensity, but may also retain the severity of a disease associated with considerable lifestyle impairment [11]. In the present study, the associations of dry eye symptoms with various psychiatric disorders (depression, anxiety, attention deficit hyperactivity disorder, and sleep disorder) were examined through a retrospective self-reporting questionnaire study.

\section{Subjects and Methods}

This study was conducted for people who complained of dry eye disease symptoms for the first time between September 2014 and February 2015. All subjects completed psychiatric and ophthalmic questionnaires.

\section{Participants}

Adults residing in the Anseong area between September 2014 and February 2015 were participated. Anseong city with 180,000 population. We asked many companies that located Anseong city for research, we have random selection among 6 companies receiving the acceptance for research. 600 adults living in the research target area were given questionnaires to answer. Among them, 502 people responded with a rate of $83.7 \%$. Subjects were excluded from the study if there was any evidence of psychotic disorder, mental retardation and neurological disorders including epilepsy. The present study was approved by the Institutional Review Board of Dankook University Hospital (DK-201402-004). All participants were interviewed personally and given a full verbal explanation and short form document with information about the study, including the study's purpose and procedure. Informed written consent was obtained from each participant before the study began.

\section{Assessments}

\section{Epidemiological questionnaire}

Epidemiological Questionnaire were consisted of questions on sex, age, socioeconomic status, and recent work achievement.

\section{The Ocular surface disease index (OSDI)}

The Ocular Surface Disease Index (OSDI ${ }^{\oplus}$ was developed to quantify the specific impact of dry eyes on vision-targeted health-related quality of life (VT-HRQ) [12]. This disease-specific questionnaire includes three subscales: ocular discomfort (OSDI-symptoms), which includes symptoms such as gritty or painful eyes; functioning (OSDI-function), which measures limitation in performance of common activities such as reading and working on a computer; and environmental triggers (OSDI-triggers), which measures the impact of environmental triggers, such as wind or drafts, on dry eye symptoms. The questions are asked with reference to a one-week recall period. Possible responses refer to the frequency of the disturbance: none of the time, some of the time, half of the time, most of the time, or all of the time. Subscale scores were computed for OSDI-symptoms, OSDI-function, and OSDI- triggers, as well as an overall averaged score. OSDI subscale scores can range from 0 to 100 , with higher scores indicating more problems or symptoms.

\section{Center for Epidemiological studies-depression scale (CES-D)}

This CES-D was developed by Radloff et al. to measure the degree of adult depression; Cho and Kim standardized this resulting in an internal reliability(Chronbach's alpha) was 0.91 [13]. Items were scored using conventional 1-2-3-4 Likert scale scores for the response categories.

\section{Korean adult attention deficit hyperactivity disorder scales (K-AADHS)}

Developed by Murphy and Barkley, based on the DSM-IV diagnostic criteria for adult ADHD; the K-AADHS is a self-report assessment. The scales, with 18 items, is proven for its validity in differentiating adults with ADHD from those without, and is designed to effectively differentiate three subtypes of ADHD: predominantly inattentive, predominantly hyperactive-impulsive, and combined hyperactive-impulsive, and inattentive. The Korean standardization has been achieved by Kim et al. The chronbach alpha of the total K-AADHS scale was 0.85 [14].

\section{The Korean occupational Stress Scale- Short Form (KOSS-SF)}

The short form of KOSS-SF, consisted of 24 items and was developed and standardized by Jang et al. [15]. These are considered to be unique and specific occupational stressors for Korean employees. They collected KOSS-SF items from the most popular job stress measurement tools such as the JCQ, ERI, NIOSH and OSI, and additive qualitative studies (depth interview). Items were scored using conventional 1-23-4 Likert scale scores for the response categories. This scale consisted of seven subscales by using factor analysis and a validation process: job demand, insufficient job control, interpersonal conflict, job insecurity, organizational system, lack of reward, and occupational climate. Internal consistency chronbach alpha scores ranged from 0.51 to 0.82 .

\section{The Korean version of Pittsburgh Sleep Quality Index (PSQI-K)}

The Pittsburgh Sleep Quality Index (PSQI) is typically used for evaluating sleep quality throughout the world, developed by Buysse and colleagues [16]. This is a self-reported questionnaire, which evaluates sleep quality during the previous month. Sohn et al. [17] analyzed the reliability and validity of the Korean version of the PSQI (PSQI-K). Cronbach's a coefficient for internal consistency of the total score of the PSQI-K was 0.84 , which shows high reliability. Sensitivity and specificity for distinguishing poor and good sleepers were 0.943 and 0.844 using the best cutoff point of 8.5. The test-retest correlation coefficient was 0.65 for the total score $(\mathrm{p}<0.001)$.

\section{The Korean version of Epworth sleepiness scale (KESS)}

The Epworth sleepiness scale (ESS) is widely used to measure the general level of daytime sleepiness, particularly among patients with sleep disordered breathing $[18,19]$. The ESS is comprised of eight questions, each asking about the subject's likelihood of dozing off or falling asleep in a particular but common situation that he/she would encounter on typical day. Cho et al. [20] developed the Korean version of the ESS (KESS), which is valid and has been evaluated for its usefulness. 
The KESS in patient groups showed good internal consistency (Cronbach's $\alpha=0.90)$ and test-retest reliability ( $r=0.78$ to 0.93 ).

\section{Statistical analysis}

The data was processed using SPSS 15.0 (Korean version). In the statistical analysis, a cross tabulation analysis was performed for an epidemiologic survey evaluation (e.g., gender). An ANCOVA test considering age and sex was used for the analysis of the OSDI, BDI, K-AADHS, PSQI, and ESS scores between both groups. A chi-square test was used to compare the frequencies among the depression group, ADHD group, and sleep problem group, which had a score of higher quartile. The odd ratio to the dry eye disease symptoms group was calculated using a logistic regression analysis. All the statistical analyses were considered statistically significant when the p-value was below 0.05 .

\section{Results}

The dry eye symptom group included 42 males (30.2\%) and 97 females (69.8\%), and the comparison group included 142 males (39.1\%) and 221 females (60.8\%). Thus, there was not a significant frequency difference between both groups $\left(\mathrm{X}^{2}=3.43, \mathrm{p}=.064\right)$. The average age of the dry eye symptom group was $45.22 \pm 11.00$ and that of the comparison group was $41.76 \pm 11.55$, resulting in a significant difference between both groups $(\mathrm{F}=9.27, \mathrm{p}=.002)$. The dry eye symptom group had a total OSDI score of $61.94 \pm 11.20$, and the comparison group had a total OSDI score of $22.79 \pm 15.20$. The results indicate that there was a significant difference between both groups $(\mathrm{F}=763.82$, $\mathrm{p}<.001)$. The dry eye symptom group had a total CES-D score of 11.70 \pm 8.14 , and the comparison group had a total CES-D score of $7.91 \pm$ 5.94 , resulting in a significant difference between both groups $(\mathrm{F}=32.43$, $\mathrm{p}<.001)$. The dry eye symptom group had a total K-AADHS score of $9.07 \pm 7.59$, and the comparison group had a total K-AADHS score of $5.69 \pm 6.32$, resulting in a significant difference between both groups $(\mathrm{F}=25.39, \mathrm{p}<.001)$. The dry eye symptom group had a total PSQI score of $6.93 \pm 3.21$, and the comparison group had a total PSQI score of $5.30 \pm 3.03$, resulting in a significant difference between both groups $(\mathrm{F}=26.03, \mathrm{p}<.001)$. The dry eye symptom group had a total ESS score of $8.11 \pm 5.03$, and the comparison group had a total ESS score of $5.74 \pm$ 4.42 , resulting in a significant difference between both groups $(\mathrm{F}=26.07$, $\mathrm{p}<.001)$ (Table 1). In regards to the dry eye symptom group with depression, there were 58 subjects $(42.3 \%)$, and the non-depression dry eye symptom group included 81 subjects (57.7\%). While for the general comparison group, the depression group included 70 subjects (19.6\%) and the non-depression group included 293 subjects (80.4\%). Thus, there was a significant frequency difference between both groups $\left(x^{2}=26.64, p<.001\right)$. For dry eye symptom group with ADHD there were 58 subjects $(41.7 \%)$, and the non-ADHD dry eye symptom group included 81 subjects (58.3\%). While for the general comparison group, the ADHD group included 71 subjects (19.9\%) and the non-ADHD group included 292 subjects $(80.1 \%)$. Thus, there was a significant frequency difference between both groups $\left(x^{2}=24.80, p<.001\right)$. For the dry eye symptom group with high job stress there were 41 subjects (30.6\%), and the low job stress dry eye symptom group included 98 subjects $(69.4 \%)$. While for the general comparison group, the high job stress group included 82 subjects $(23.4 \%)$ and the low job stress group included 281 subjects $(76.6 \%)$. Thus, there was not a significant frequency difference between both groups $\left(\mathrm{x}^{2}=2.63, \mathrm{p}<.001\right)$. For the dry eye symptom group with sleep problems there were 48 subjects (37.2\%), and the non-sleep problem dry eye symptom group included 91 subjects $(62.8 \%)$. While for the general comparison group, the sleep

\begin{tabular}{|l|c|c|c|c|}
\hline Rating Scale & $\begin{array}{c}\text { Dry eye disease } \\
\text { group (n=139) } \\
\text { Mean } \pm \text { S.D }\end{array}$ & $\begin{array}{c}\text { Comparison } \\
\text { group (n=363) } \\
\text { Mean } \pm \text { S.D }\end{array}$ & F or $\mathbf{x}^{2}$ & p value \\
\hline Age $^{\text {a }}$ & $45.22 \pm 11.00$ & $41.76 \pm 11.55$ & 9.27 & .002 \\
\hline Sex & $42(30.2 \%)$ & $142(39.1 \%)$ & & .064 \\
\hline Male & $97(69.8 \%)$ & $221(60.9 \%)$ & & \\
\hline Female & & & 1.95 & .378 \\
\hline $\begin{array}{l}\text { Recent work } \\
\text { achievement (N,\%) }\end{array}$ & $56(40.3 \%)$ & $142(39.1 \%)$ & & \\
\hline High & $82(59.0 \%)$ & $211(58.1 \%)$ & & \\
\hline Middle & $1(0.7 \%)$ & $10(2.8 \%)$ & & \\
\hline Low & & & \\
\hline
\end{tabular}

Table 1: Epidemiological Characteristics between Dry Eye Disease Group and Comparison Group. Note: independent t test, ${ }^{a}$ or chi-square test, ${ }^{b} \mathrm{p}$ value $<.05$.

\begin{tabular}{|l|c|c|c|c|}
\hline Rating Scale & $\begin{array}{c}\text { Dry eye disease } \\
\text { group (n=139) } \\
\text { Mean } \pm \text { S.D }\end{array}$ & $\begin{array}{c}\text { Comparison group } \\
\mathbf{( n = 3 6 3 )} \\
\text { Mean } \pm \text { S.D }\end{array}$ & F & p value \\
\hline OSDI & $61.94 \pm 11.20$ & $22.78 \pm 15.20$ & 763.82 & $<.001$ \\
\hline CES-D & $11.70 \pm 8.14$ & $7.91 \pm 5.94$ & 32.43 & $<.001$ \\
\hline K-AADHS & $9.07 \pm 7.59$ & $5.69 \pm 6.32$ & 25.39 & $<.001$ \\
\hline Inattention & $4.83 \pm 4.22$ & $3.21 \pm 3.43$ & 19.40 & $<.001$ \\
\hline Hyperactivity & $4.23 \pm 3.83$ & $2.48 \pm 3.29$ & 26.01 & $<.001$ \\
\hline Job Stress & $51.58 \pm 12.12$ & $49.87 \pm 11.36$ & 2.10 & .148 \\
\hline PSQI & $6.93 \pm 3.21$ & $5.30 \pm 3.03$ & 26.03 & $<.001$ \\
\hline ESS & $8.11 \pm 5.03$ & $5.74 \pm 4.42$ & 26.07 & $<.001$ \\
\hline
\end{tabular}

Table 2: Suicide idea, depression, anxiety, ADHD, self-esteem, impulsivity between Dry Eye Disease Group and Comparison Group. Note: OSDI; the Ocular Surface Disease Index, CES-D; Center for Epidemiological Studies-Depression Scale, K-AADHS; Korean Adult Attention Deficit Hyperactivity Disorder Scales, and PSQI-K; The Korean version of Pittsburgh Sleep Quality Index. p value <.05.

problem group included 70 subjects $(20.7 \%)$ and the non-sleep problem group included 293 subjects (79.3\%). Thus, there was a significant frequency difference between both groups $\left(x^{2}=13.46, p<.001\right)$. For the dry eye symptom group with ESS there were 58 subjects (42.3\%), and the non-ESS dry eye symptom group included 81 subjects (57.7\%). While for the general comparison group, the ESS group included 77 subjects (21.8\%) and the non-ESS group included 286 subjects (78.2\%). Thus, there was a significant frequency difference between both groups $\left(\mathrm{x}^{2}=20.99, \mathrm{p}<.001\right)$ (Table 2). In the logistic regression model of the dry eye symptom group and the comparison group, the relative risk of depression was 1.75 times higher (confidence interval 1.03-2.97), which showed a statistical significance $\left(\mathrm{x}^{2}=4.20, \mathrm{p}=.04\right)$. The relative risk of ADHD was 2.18 times higher (confidence interval 1.33-3.57), which also showed a statistical significance $\left(x^{2}=9.49, \mathrm{p}=.00\right)$ (Table 3$)$.

\section{Discussion}

A previous study reported that females showed a significantly higher prevalence rate of dry eye disease than males [21]. In the present study, the number of female patients was also larger than that of males. As well as an objective ophthalmological examination, the evaluation of subjective symptoms was a crucial finding on the clinical diagnosis of dry eye disease. Many studies reported that there were difficulties in the treatment of dry eye disease patients because objective clinical findings were inconsistent with the symptoms that patients complained of [22] The OSDI value is an examination that tests the symptoms patients suffering from dry eye disease complain of, but the OSDI value, the common representative evaluation tool of dry eye disease, could be inconsistent with the degree of dry eye disease [23]. In the present study, the OSDI, CES-D, KOSS, K-AADHS, PSQI, ESS values based on a psychiatric questionnaire were compared between the dry eye 


\begin{tabular}{|c|c|c|c|c|}
\hline Rating Scale & $\begin{array}{c}\text { Dry eye disease } \\
\text { group (n=139) } \\
\mathbf{N}(\%)\end{array}$ & $\begin{array}{c}\text { Comparison } \\
\text { group(n=363) } \\
\mathbf{N}(\mathbf{m})\end{array}$ & $X^{2}$ & p value \\
\hline CES-D (>=21) & $58(42.3 \%)$ & $70(19.6 \%)$ & 26.64 & $<.001$ \\
\hline K-AADHS (>=19) & $58(41.7 \%)$ & $71(19.9 \%)$ & 24.80 & $<.001$ \\
\hline KOSS-SF & $41(30.6 \%)$ & $82(23.4 \%)$ & 2.63 & $<.001$ \\
\hline PSQI & $48(37.2 \%)$ & $70(20.7 \%)$ & 13.46 & $<.001$ \\
\hline ESS (>=10) & $58(42.3 \%)$ & $77(21.8 \%)$ & 20.99 & $<.001$ \\
\hline
\end{tabular}

Table 3: Depression, ADHD, Job Stress, Sleep Disturbance, Daytime Sleepiness between Dry Eye Disease Group and Comparison Group. Note: OSDI; the Ocula Surface Disease Index, CES-D; Center for Epidemiological Studies-Depression Scale, K-AADHS; Korean Adult Attention Deficit Hyperactivity Disorder Scales, and PSQI-K; The Korean version of Pittsburgh Sleep Quality Index. p value $<.05$

\begin{tabular}{|l|c|c|c|c|c|}
\hline & $\begin{array}{c}\text { Parameter } \\
\text { estimate }\end{array}$ & $\begin{array}{c}\text { Standard } \\
\text { error }\end{array}$ & $\begin{array}{c}\text { Chi- } \\
\text { square }\end{array}$ & p Value & Odds ratio \\
\hline Sex & -.39 & .24 & 2.74 & .10 & $.67(.42-1.05)$ \\
\hline CES-D & .56 & .27 & 4.20 & .04 & $1.75(1.03-2.97)$ \\
\hline K-AADHS & .78 & .25 & 9.49 & .00 & $2.18(1.33-3.57)$ \\
\hline KOSS & .25 & .26 & .92 & .34 & $1.28(.77-2.13)$ \\
\hline PSQI & .47 & .26 & 3.39 & .07 & $1.60(.97-2.65)$ \\
\hline
\end{tabular}

Table 4: Parameter Estimates for Logistic Model of Dry Eye Disease Group and Comparison Group. Note: OSDI; the Ocular Surface Disease Index, CES-D Center for Epidemiological Studies-Depression Scale, K-AADHS; Korean Adult Attention Deficit Hyperactivity Disorder Scales, and PSQI-K; The Korean version of Pittsburgh Sleep Quality Index. p value <.05.

symptoms group and the comparison group; and the dry eye symptoms group had significantly higher OSDI, CES-D, KOSS, K-AADHS, PSQI, ESS values (Table 4). The analysis of the odd ratio, depending on the presence of dry eye symptoms using the regression analysis, showed that the CES-D, K-AADHS values increased the odd ratio of dry eye symptoms, respectively $(\mathrm{p}=0.04$ and $\mathrm{p}<0.001)$. This indicates associations between dry eye symptoms, depression, and ADHD. In the present study, the DES group had a high depression score, and this is consistent with results of previous research [5,6,8-10]. However, there has been no study on the association between DES and ADHD, but was attempted in the present study for the first time. The results indicated that the DES group had a significantly higher ADHD scale score than the control group, and ADHD showed a significant correlation with DES even after a regression analysis with sex/age adjustment. It has been a few reported that depression and anxiety, common internalized symptoms are related with the dry eye syndrome. However, there has been no report on the relationship of ADHD or ADHD symptoms, common externalized symptoms with dry eye syndrome. ADHD is a common behavioral disorder usually has its onset in early childhood, more rarely in adolescence. When ADHD persists into adulthood, the disorder may be of mild intensity, but may also retain the severity of a disease associated with considerable lifestyle impairment. We suspected that its inattention and impulsivity symptoms may be affect the individual eye care and maintenance. It caused to stressful state. Stress is a common etiology of dry eye syndrome [24,25]. The results of the present study showed that ADHD as well as depression was the risk factor of DES. Therefore, it is thought that the treatment of dry eye disease could be affected by cognitive and behavioral characteristics (e.g., inattention and impulsiveness) as well as psychological factors (e.g., depression). The limitations of this study are as follows. First, the severity of the dry eye symptoms group was very severe than the general population because all subjects worked at an industrial factory. Second, for the general control group, a questionnaire evaluation regarding dry eye disease was performed, but physical an ophthalmic examination was not performed. Third, since the study was based on a self-report type questionnaire difference in the cognitive ability of participants could affect the result of the study. To minimize this effect, sex, age, socioeconomic status or work achievement level needs to be used as a control factor during analysis, but sufficient relevant data could not be collected. Especially the previous studies reported that females showed significantly higher prevalence rate of depression than males. Though not statistically significantly, our study showed the number of female patients was larger than that of males relatively. Fourth, it was a cross-sectional research, and thus the causal relationship between both groups could not be suggested. Only the relationships of dry eye disease with depression symptoms, ADHD, and sleep problems were suggested. Fifth, this study examined participants residing in only one local community. The area where the data of the patient group and the control group were obtained was a rural area with a population of about 200,000 , respectively. Thus, it was difficult to reflect the characteristics of metropolitan cities. In the future, a well-structured patient/control group study needs to be performed by correcting for gender, age, and area distribution.

\section{References}

1. Lemp MA, Foulks GN (2007) the definition and classification of dry eye disease Ocular Surf 5: 75-92.

2. Sullivan BD, Crews LA, Messmer EM, Foulks GN, Nichols KK, et al. (2014) Correlations between commonly used objective signs and symptoms for the diagnosis of dry eye disease: clinical implications. Acta ophthalmologica 92 161-166.

3. Smith JA (2007) The epidemiology of dry eye disease. Acta Ophthalmologica Scandinavica 85: s240

4. Van der Vaart R, Weaver MA, Lefebvre C, Davis RM (2015) The Association between Dry Eye Disease and Depression and Anxiety in a Large PopulationBased Study. Am J Ophthalmol 159: 470-474.

5. Labbé A, Wang YX, Jie Y, Baudouin C, Jonas JB, et al. (2013) Dry eye disease dry eye symptoms and depression: the Beijing Eye Study. Br J Ophthalmol 97 1399-1403.

6. Li M, Gong L, Sun X, Chapin WJ (2011) Anxiety and depression in patients with dry eye syndrome. Curr Eye Res 36: 1-7.

7. Wen W, Wu Y, Chen Y, Yan M, Xiao Z, et al. (2012) Dry eye disease in patients with depressive and anxiety disorders in Shanghai. Cornea 31: 686-692.

8. Galor A, Feuer W, Lee DJ, Florez H, Faler AL, et al. (2012) Depression, posttraumatic stress disorder, and dry eye syndrome: a study utilizing the national United States Veterans Affairs administrative database. Am J Ophthalmol 154 340-346.

9. Na KS, Han K, Park YG, Na C, Joo CK (2015) Depression, Stress, Quality of Life, and Dry Eye Disease in Korean Women: A Population-Based Study. Cornea 34: 733-738.

10. Fernandez CA, Galor A, Arheart KL, Musselman DL, Venincasa VD, et al. (2013) Dry eye syndrome, posttraumatic stress disorder, and depression in an older male veteran population. Invest Ophthalmol Vis Sci 54: 3666-3772.

11. Mannuzza S, Klein RG, Bessler A, Malloy P, LaPadula M (1993) Adult outcome of hyperactive boys. Educational achievement, occupational rank, and psychiatric status. Arch Gen Psychiatry 50: 565-576.

12. Schiffman RM, Christianson MD, Jacobsen G, Hirsch JD, Reis BL (2000) Reliability and validity of the Ocular Surface Disease Index. Arch Ophthalmo 118: $615-621$

13. Cho MJ, Kim KH (1993) Diagnostic validity of the CES-D (Korean version) in the assessment of DSM-III-R major depression. J Korean Neuropsychiatr Assoc 32: 381-399.

14. Kim EJ (2003) The validation of Korean Adult ADHD Scale. Korean J Clinical Psychology 22: 897-911.

15. Chang SJ, Koh SB, Kang DM, Kim SA, Kang MG, et al. (2005) Developing an Occupational Stress Scale for Korean Employees. Korean J Occup Environ Med 17: 297-317. 
Citation: Cho KJ, Kim HK, Lim MH, Baek HS, Yang YA, et al. (2015) Depression, ADHD, Job Stress, and Sleep Problems with Dry Eye Disease in Korea. J Psychiatry 18: 331 doi:10.4172/2378-5756.1000331

Page 5 of 5

16. Buysse DJ, Reynolds CF 3rd, Monk TH, Berman SR, Kupfer DJ (1989) The Pittsburgh Sleep Quality Index: a new instrument for psychiatric practice and research. Psychiatry Res 28: 193-213.

17. Sohn SI, Kim DH, Lee MY, Cho YW (2012) the reliability and validity of the Korean version of the Pittsburgh Sleep Quality Index. Sleep Breath 16: 803-812.

18. Johns MW (1993) Daytime sleepiness, snoring, and obstructive sleep apnea. The Epworth sleepiness scale. Chest 103: 30-36.

19. Johns MW (1991) A new method for measuring daytime sleepiness: the Epworth sleepiness scale. Sleep 14: 540-545.

20. Cho YW, Lee JH, Son HK, Lee SH, Shin C, et al. (2011) The reliability and validity of the Korean version of the Epworth sleepiness scale. Sleep Breath 15: 377-384.

21. Moss SE, Klein R, Klein BE (2000) Prevalence of and risk factors for dry eye syndrome. Arch Ophthalmol 118: 1264-1268.
22. Begley CG, Chalmers RL, Abetz L, Venkataraman K, Mertzanis P, et al. (2003) The relationship between habitual patient-reported symptoms and clinical signs among patients with dry eye of varying severity. Invest Ophthalmol Vis Sci 44 4753-4761.

23. Nichols KK, Nichols JJ, Mitchell GL (2004) The lack of association between signs and symptoms in patients with dry eye disease. Cornea 23: 762-770.

24. Mizuno Y, Yamada M, Miyake Y (2010) Dry Eye Survey Group of the National Hospital Organization of Japan. Association between clinical diagnostic tests and health-related quality of life surveys in patients with dry eye syndrome. Jpn J Ophthalmol 54: 259-265.

25. Sullivan BD, Whitmer D, Nichols KK, Tomlinson A, Foulks GN, et al. (2010) An objective approach to dry eye disease severity. Invest Ophthalmol Vis Sci 51 6125-6130. 